\title{
Rare malformations associated with partial anomalous pulmonary venous return: a cadaveric case report
}

\author{
S. Silawal, T. Essing, C. Schaupp, G. Schulze-Tanzil \\ Institute of Anatomy and Cell Biology, Paracelsus Medical University, Nuremberg, Germany \\ [Received: 13 January 2021; Accepted: 11 February 2021; Early publication date: 1 March 2021]
}

\begin{abstract}
A unique partial anomalous pulmonary venous return in combination with other rare malformations such as annular pancreas and a persistent umbilical vein was discovered in a female Caucasian cadaver during an anatomical dissection at the Paracelsus Medical University in Nuremberg, Germany. The pulmonary anomaly comprised the aberrant left superior pulmonary vein connecting the superior lobe of the left lung with the left brachiocephalic vein resulting in a left to right shunt. An annular pancreas without any signs causing duodenal compression was additionally found. To complete the constellation of malformations, a persistent umbilical vein within the round ligament fissure of the liver was also observed, connecting to an inferior branch of the extrahepatic left ramus of the portal vein. This rare constellation of malformations has been illustrated and thoroughly discussed with the currently available literature to develop a hypothesis for the genetic and developmental background. (Folia Morphol 2022; 81, 2: 526-531)
\end{abstract}

Key words: partial anomalous pulmonary venous return, patent umbilical vein, annular pancreas

\section{INTRODUCTION}

Partial anomalous pulmonary venous return (PAPVR) is a cardiovascular congenital anomaly characterised by a partial alteration in the venous connection of the oxygenated blood of the pulmonary vein to the left atrium resulting in a left to right shunt. The venous drainage point of return of the partial anomalous pulmonary vein can vary in its site; occurring most frequently supracardial (63\%), followed by the cardial $(20 \%)$, mixed $(11 \%)$ and infracardial (6\%) variants [14]. In contrast to the total anomalous pulmonary venous return (TAPVR), which is a deadly anomaly, PAPVR does not necessarily demonstrate clinical symptoms. The actual prevalence could therefore be even higher than the reported $0.4-0.7 \%$ of adults shown in autopsy cases [12]. Although the literature shows that right sided PAPVR is more frequent $[1,7,21]$, a study with computed tomography images from 29 adults showed a connection of the superior left pulmonary vein into the left persistent vertical vein in $79 \%$ of their studied cases [11].

Partial anomalous pulmonary venous return often exists in combination with other multiple congenital anomalies [21]. An interesting clinical case report about PAPVR with persistent left superior vena cava, 'bovine arch' aortic branching, tracheal diverticulum, aberrant lung fissure and an annular pancreas (AP) has been published [17]. We introduce a similar constellation; however, in a cadaveric gross anatomy, where we could find PAPVR combined with other 

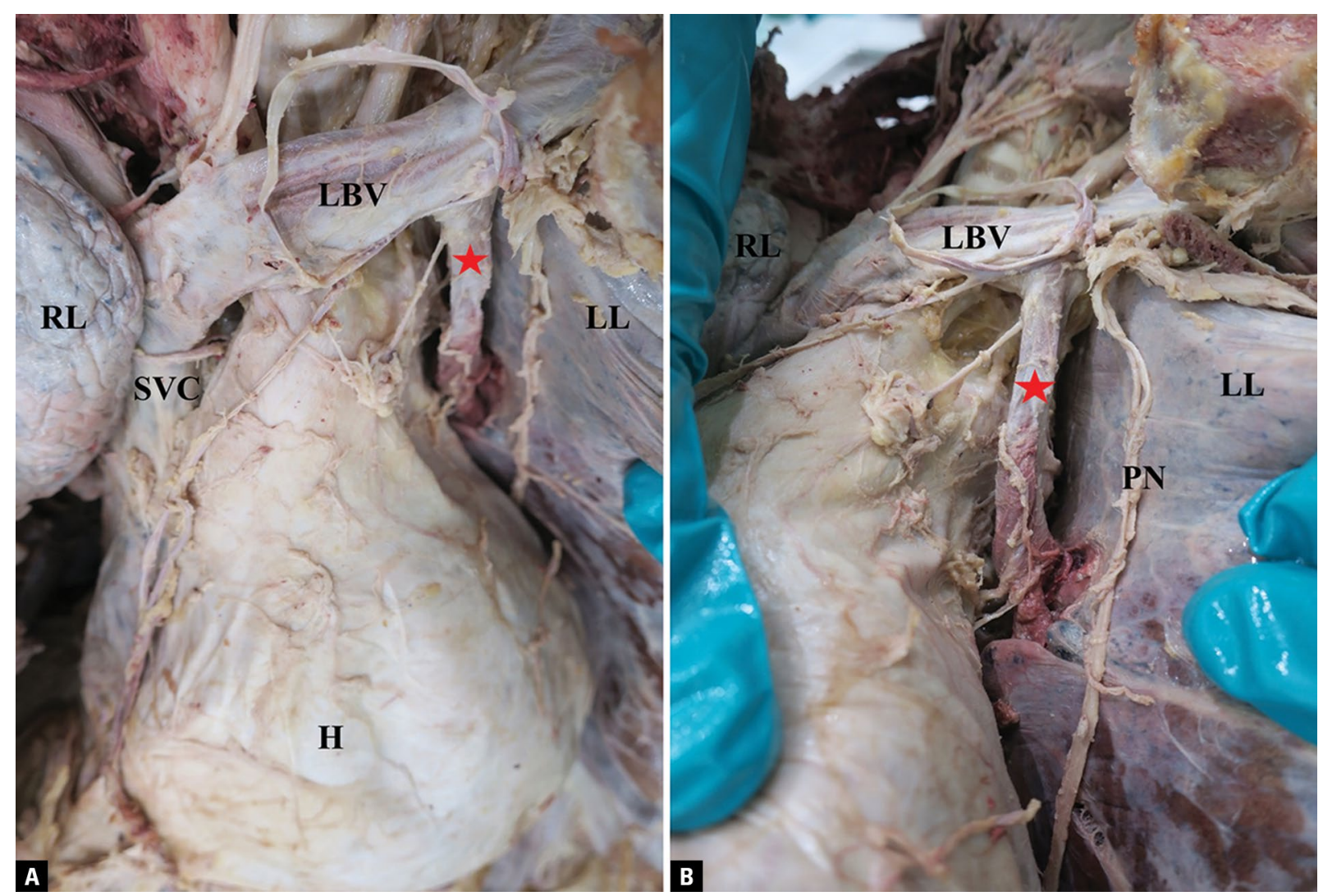

Figure 1. A. Anterior view on the mediastinum; B. Anterolateral view on the mediastinum; RL — right lung; $\mathrm{LL}$ — left lung; $\mathrm{H}$ - heart (covered by the parietal pericardium); LBV — left brachiocephalic vein; SVC — superior vena cava; PN — phrenic nerve; red star — left superior pulmonary vein with anomalous return.

multiple congenital anomalies (MCA) such as AP and patent umbilical vein (PUV) with connection to the left portal vein of the liver.

Annular pancreas is a congenital anomaly in which the pancreas either completely or partially encircles the descending portion of duodenum. This phenomenon can obstruct the gastrointestinal lumen, occasionally leading to duodenal stenosis [10].

This constellation will be discussed in detail with the available literature of comparable cases for specific genetic mutations being associated with the individual variations observed in the described case and an effort will be made to find a common ground to explain the coexistence of the MCA.

\section{CASE REPORT}

The formalin-fixed cadaver of an 88-year-old female body donor came from the body donor system of the LMU, Munich, Germany. Photos were taken using a Canon Camera (G9 X, Tokyo, Japan) and measurements were taken using a digital calliper (Ovibell GmbH, Mühlheim, Germany). Haematoxylin and eosin staining was performed in the Institute of Pathology, General Hospital Nuremberg to verify the observation. The histological image was taken using a DM1000 LED light microscope (Leica, Wetzlar, Germany). Literature research related to the anomalies was undertaken using Medline and Google Scholar by searching mesh terms for genetic mutations such as: partial anomalous, persistent right umbilical vein, annular pancreas and persistent umbilical vein.

\section{Aberrant pulmonary vein}

The aberrant/displaced superior left pulmonary vein measuring $63 \mathrm{~mm}$ in length and $6.2 \mathrm{~mm}$ in diameter drained from the left superior lobe through the pulmonary hilum into the left brachiocephalic vein (Fig. 1A, B). The $37.3 \mathrm{~mm}$ long and $12.4 \mathrm{~mm}$ thick left brachiocephalic vein joined together with the right brachiocephalic vein to form the superior vena cava, finally draining into the right atrium. However, an additional venal tributary from the lowest portion (segment $\mathrm{V}$ ) of the left superior lobe drained into the left inferior pulmonary vein from the inferior lobe of the left lung (Fig. 2A, B) entering together 

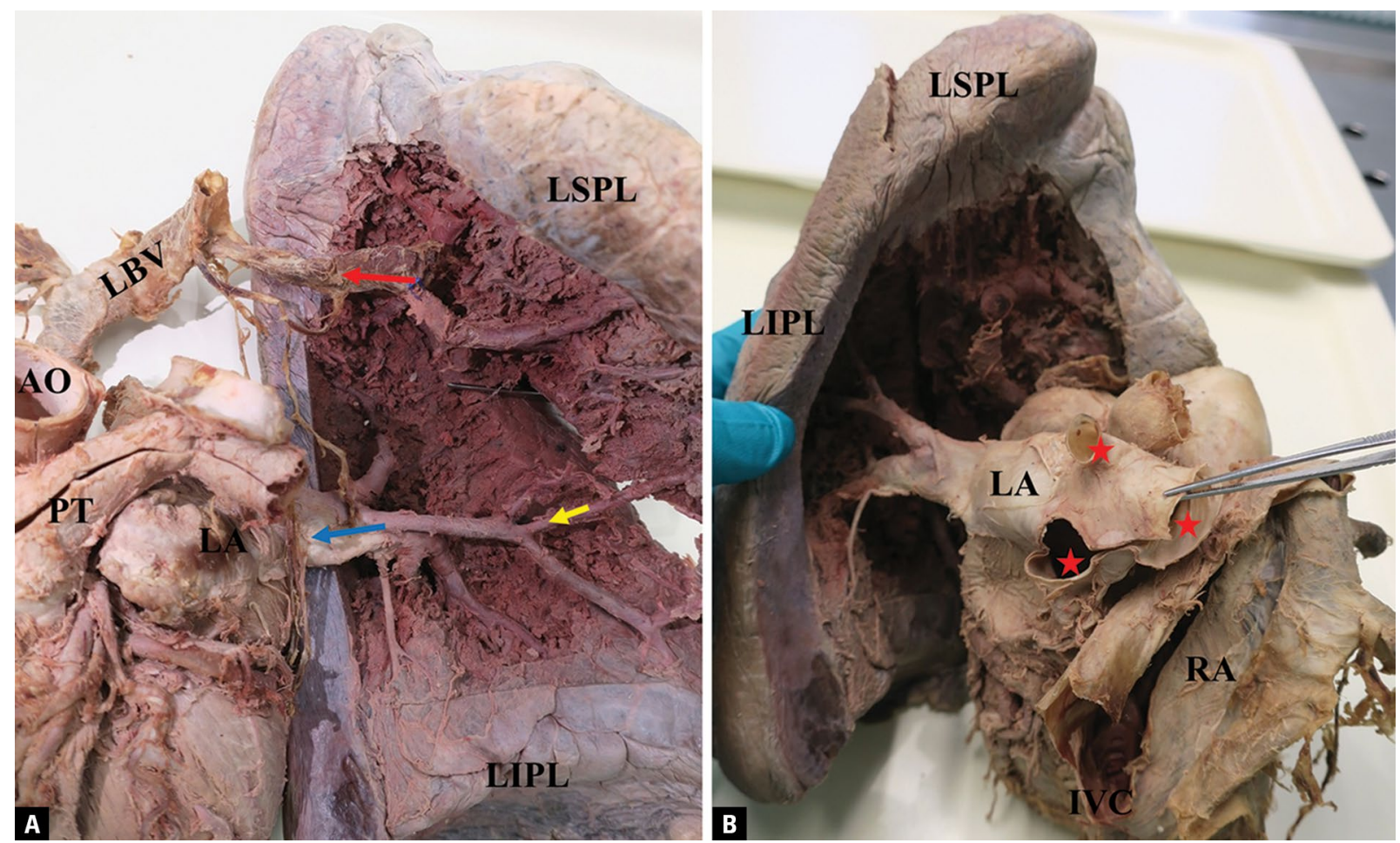

Figure 2. A. Anterior view after removal of pulmonary arteries, bronchial pathways and partially, of lung parenchyma on the mediastinal side of the left lung; B. Posterior view. The atrial wall between entrance of superior and inferior vein has been cut to get the view into the interior of the left atrium. Likewise, the atrial wall between entrance of superior vena cava and inferior vena cava has been cut to get the view into the interior of the right atrium; LIPL — left inferior pulmonary lobe; LSPL — left superior pulmonary lobe; LBV — left brachiocephalic vein; AO — aorta; PT — pulmonary trunk; LA — left atrium; RA — right atrium; IVC — inferior vena cava; red arrow — left superior pulmonary vein (anomalous); blue arrow — left inferior pulmonary vein (regular); yellow arrow — venal tributary from the lowest portion (segment V) of the left superior lobe draining into the left inferior pulmonary vein; red stars — right pulmonary veins after resection.

as a single left entrance into the left atrium. On the other side there were three separate entries, instead of two, into the left atrium from the three lobes of the right lung (Fig. 2B). The left and right lungs were of normal size and both possessed a regular oblique fissure.

Neither any irregularity in size of the heart chambers nor in any septation of the heart could be observed. The coronary sinus had no connection to the variant vein and was not enlarged.

\section{Annular pancreas}

The pancreas formed a complete circular ring surrounding the descending part of the duodenum (Fig. 3). The narrowest part of the pancreatic ring measured $8 \mathrm{~mm}$ and was located lateral on the right. The anterior part measured $17 \mathrm{~mm}$ and the posterior part was $13 \mathrm{~mm}$. The duct system of the pancreas was regular. The common bile duct ended at the descending part of the duodenum just above the AP. It showed no sign of compression, neither of the duodenum nor the common bile duct.

\section{Persistent umbilical vein and connection to the portal vein}

The persistent umbilical vein had a lumen, which was $<1 \mathrm{~mm}$ with a very thick wall of $4 \mathrm{~mm}$ (Fig. 4A). Haematoxylin and eosin staining of this vein could verify this observation (Fig. 4B). Interestingly, a connection between this lumen structure and the inferior branch of the extra hepatic left portal vein running in the round ligament fissure of the liver was seen (Fig. 5). Histologically, the thick wall of the persistent umbilical vein consisted of several layers of smooth muscle cells. The inner and outermost layer contained more longitudinally aligned muscle cell bundles and the layer between them had a more oblique orientation. The lumen was lined by flattened cells like an endothelium (Fig. 4B).

\section{DISCUSSION}

Systematic analyses concerning the frequencies of aberrant pulmonary veins are rare. Among 140 lung resection surgery patients, 23 variations were 


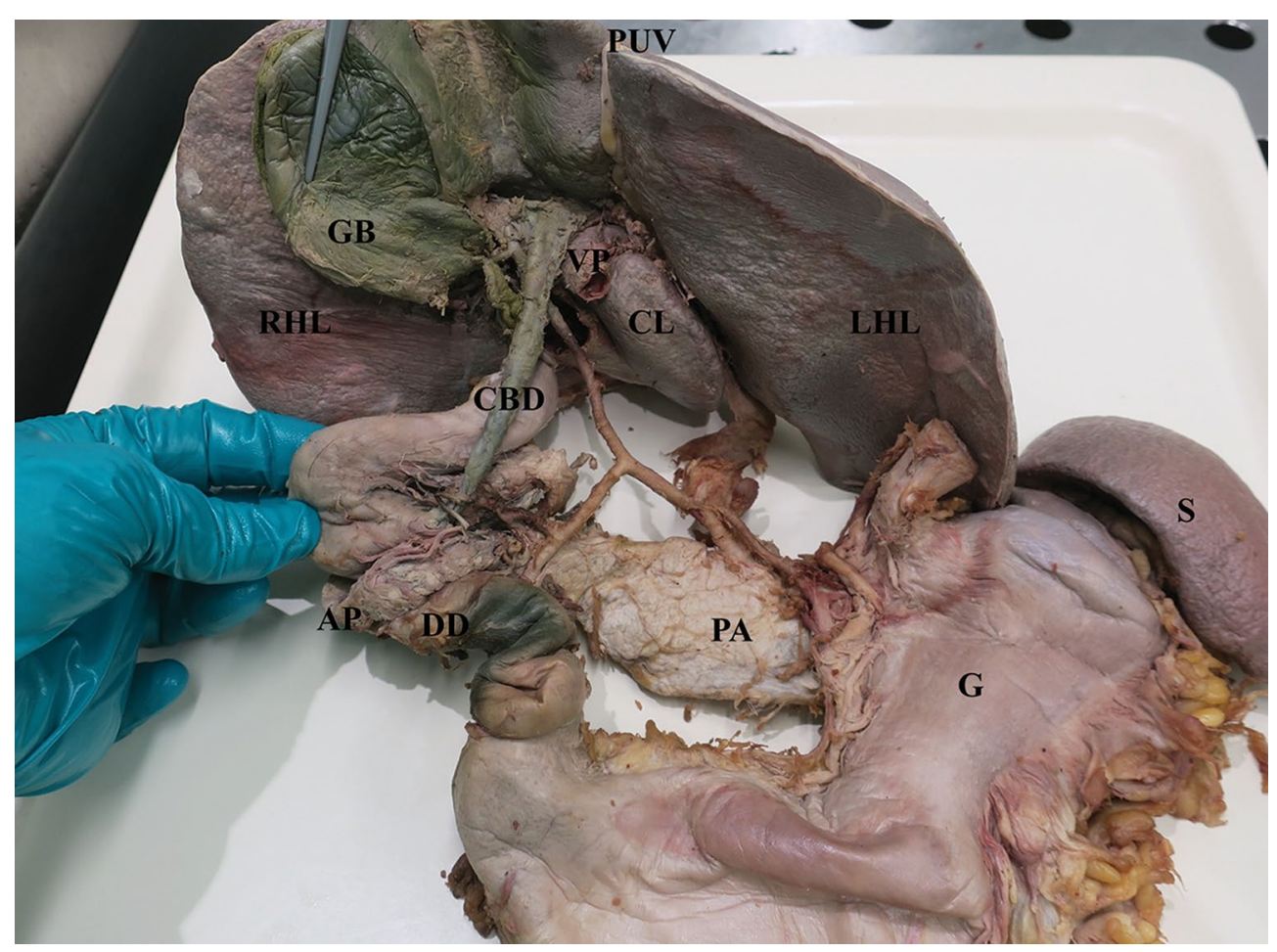

Figure 3. Dorsal view of the liver. Duodenum has been turned upside down to view common bile duct (CBD) in full length and its relationship to annular pancreas (AP); LHL — left hepatic lobe; $\mathrm{RHL}$ — right hepatic lobe; $\mathrm{CL}$ — caudate lobe; PA — pancreas; VP — portal vein; PUV — persistent umbilical vein; GB — gallbladder; DD — duodenum; G — gaster; $\mathrm{S}$ - spleen.
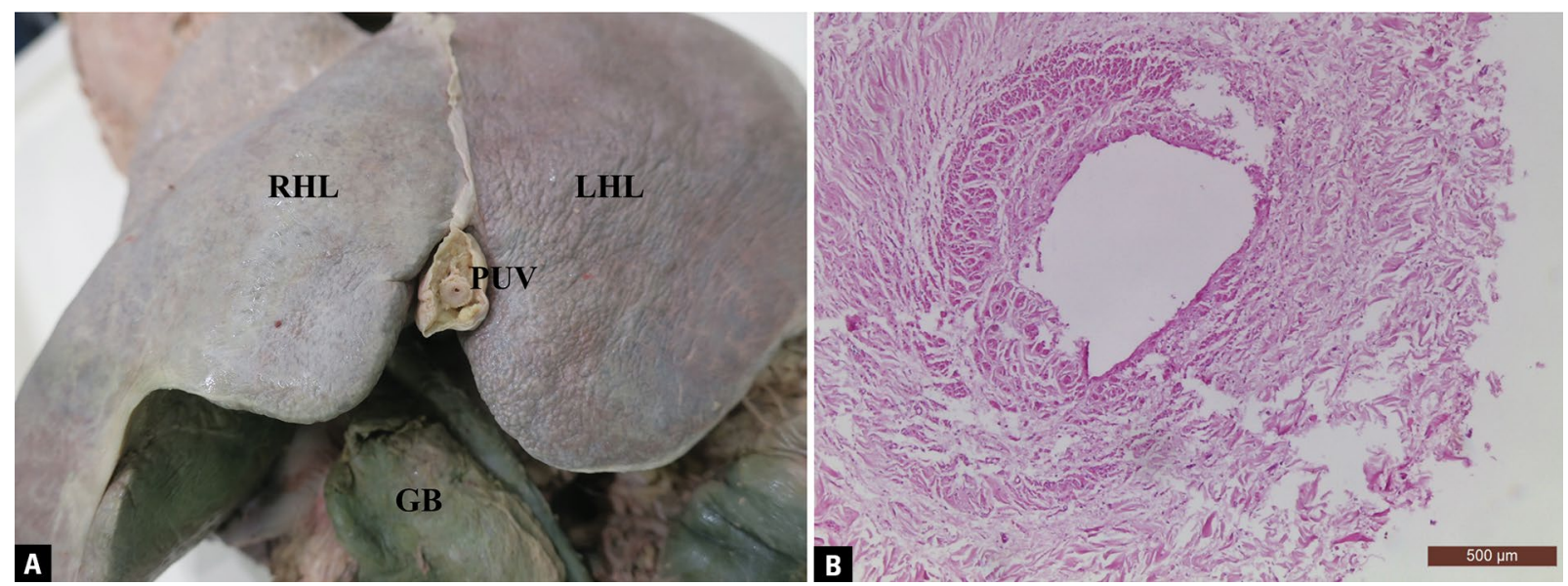

Figure 4. A. Inferior view of the liver; LHL — left hepatic lobe; RHL — left hepatic lobe; PUV — persistent umbilical vein; GB — gallbladder; B. Haematoxylin and eosin staining of persistent umbilical vein.

found [22], but no case was comparable with the variation presented here. According to the different types of PAPVR mentioned in the introduction section, our case report represents supracardial type where oxygenated blood from the left lung is directly released into the left brachiocephalic vein building a left to right shunt and subsequently collecting the mixed blood into the superior cava vein. Many of the affected patients do not present evident clinical impairments under normal conditions. However, in circumstances such as thorax surgery this large diameter vein anomaly can present a high risk. Studies have reported that superior pulmonary vein [18] or even the left inferior pulmonary vein [2] joining the 


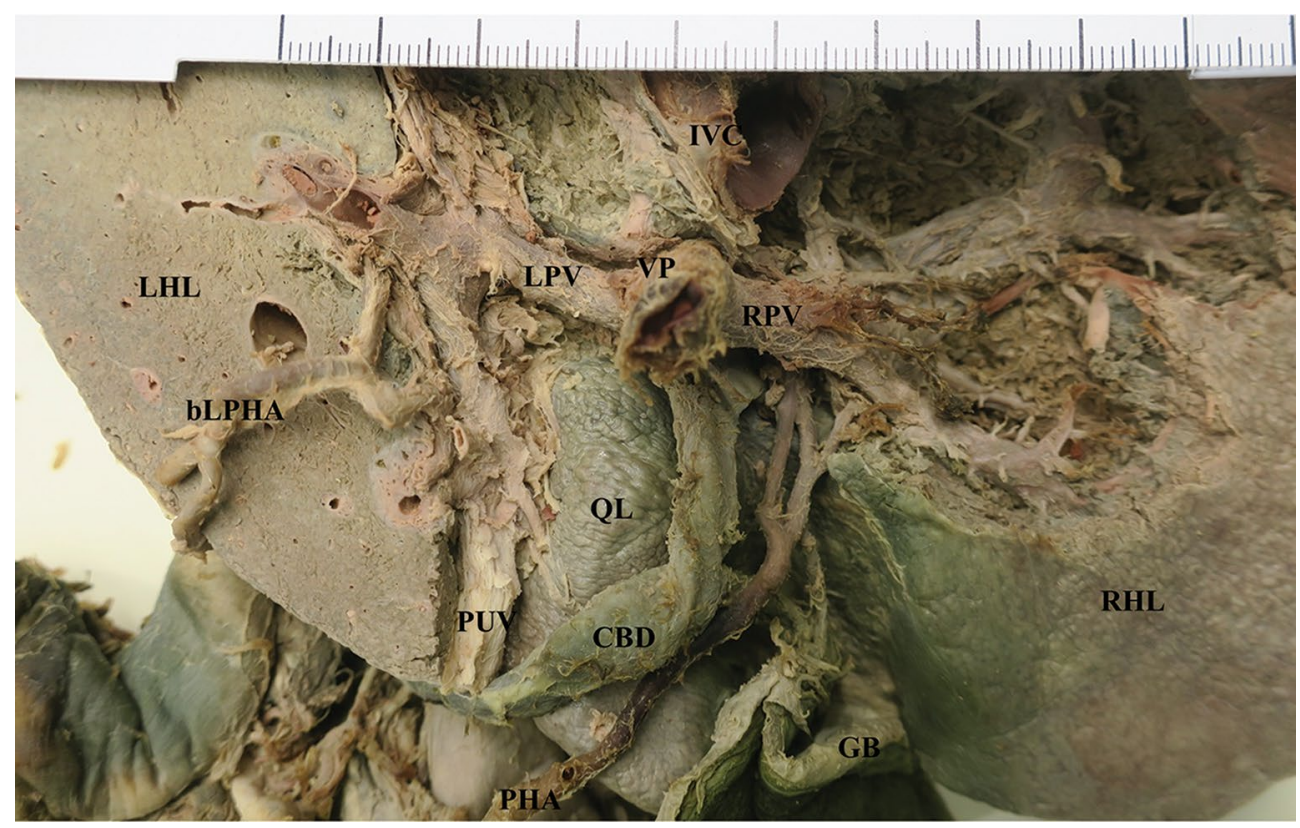

Figure 5. Dorsal view of the liver. Caudate lobe has been removed. Partial resection of segment II and III has been performed. Left proper hepatic artery has been cut and flipped aside; LHL — left hepatic lobe; RHL — left hepatic lobe; $\mathrm{OL}$ — quadrate lobe; PUV — persistent umbilical vein; GB — gallbladder; PHA — proper hepatic artery; bLPHA — branch of the left proper hepatic artery; RPV — right portal vein; LPV — left portal vein; VP — portal vein; IVC — inferior vena cava; CBD — common bile duct.

left brachiocephalic vein have been detected during clinical examination. Additional clinical relevance of this variation was observed during insertion of central venous catheter $[5,15]$. The aforementioned anomalies have been associated with genetic mutations. However, only few candidate genes are known so far. A missense gene mutation of bone morphogenetic protein receptor II could be detected in a case of anomalous unilateral single pulmonary vein [16]. In addition, a phenylalanine-to-leucine substitution that adversely affects Semaphorin $3 d$ has been identified as a putative crucial pulmonary venous patterning cue [6]. A more severe version of this anomaly is the TAPVR, which can be lethal if not corrected at an early stage. A genetic mutation in the centromeric region of chromosome 4, 4p13-q12 has been defined as a candidate for both familial and sporadic cases of TAPVR [4]. A family case of TAPVR has been reported, where a father who underwent surgical correction had 2 children with TAPVR. This supports the hypothesis of a genetic transfer pattern on the development of this anomaly [20].

Interestingly, rare variations including those of pulmonary veins are often combined with other MCA. A combination of AP with malformations of the lung [17] has already been described in a clinical setting. Even though half of the cases are asymptomatic until the third to fifth decade [10], AP presents the risk of duodenal stenosis [23]. The sonic hedgehog signalling pathway has been implicated in the development of AP [8]. Specific involvement of sonic hedgehog in mouse embryonic lung development, growth and morphogenesis has already been proven $[3,9]$, but no association with the development of PAPVR has been confirmed yet. Also, chromosome $1 \mathrm{p} 36$ deletion syndrome has been implicated in the development of AP [19].

Additionally, a persistent umbilical vein was observed in our case report. The connection of the left umbilical vein into the left portal vein during the embryological development is common. A recent study observed that 56 out of 58 embryos of gestational age 5-7 weeks showed the left sided umbilical vein draining into the left portal vein, which usually closes postnatally [13]. The closing of the umbilical vein can either be due to obliteration or simply due to collapse of the vein. This patent umbilical vein can provide access to the liver for a hepatoportography as a superior approach to diagnosis in liver disease [24]. However, recanalization of the umbilical vein is also associated with cirrhotic or non-cirrhotic portal hypertension. In our case, no macroscopic alteration of the liver could be detected. 


\section{CONCLUSIONS}

Even though we could not prove a common genetic mutation for the constellation of the mentioned anomalies, we propose that genomic sequencing in clinical settings with this constellation of anomalies could possibly help to find the genetic common ground and provide an approach in understanding the aetiology. A regular collection of tissue sample before the fixation of the cadavers could make it possible to perform genome sequencing in case of such diagnosis. However, this review should also help to highlight the cadaveric approach to define the anomaly constellation and help surgeons, radiologists and other clinicians to consider the possibility of such a combination of anatomical variations in their setting.

\section{Acknowledgements}

We are very thankful to the body donors for their contribution to the academic teaching and research field of anatomy. We would also like to thank the Institute of Pathology, General Hospital Nuremberg for performing the haematoxylin and eosin staining required for the study. Finally, special thanks to Ms. Laura Seidel for proofreading this article.

\section{Conflict of interest: None declared}

\section{REFERENCES}

1. Ammash NM, Seward JB, Warnes CA, et al. Partial anomalous pulmonary venous connection: diagnosis by transesophageal echocardiography. J Am Coll Cardiol. 1997; 29(6): 1351-1358, doi: 10.1016/s0735-1097(97)82758-1, indexed in Pubmed: 9137235.

2. Basalus MWZ, Said SAM, Stassen CM, et al. Clinical and diagnostic features of partially anomalous pulmonary venous connection in an adult female patient: a case report and review of the literature. Neth Heart J. 2011; 19(5): 256-258, doi: 10.1007/s12471-011-0101-9, indexed in Pubmed: 21487753.

3. Bellusci S, Furuta Y, Rush MG, et al. Involvement of Sonic hedgehog (Shh) in mouse embryonic lung growth and morphogenesis. Development. 1997; 124(1): 53-63, doi: 10.1242/dev.124.1.53, indexed in Pubmed: 9006067.

4. Bleyl S, Nelson L, Odelberg SJ, et al. A gene for familial total anomalous pulmonary venous return maps to chromosome 4p13-q12. Am J Hum Genet. 1995; 56(2): 408-415, indexed in Pubmed: 7847375.

5. Chintu MR, Chinnappa S, Bhandari S. Aberrant positioning of a central venous dialysis catheter to reveal a left-sided partial anomalous pulmonary venous connection. Vasc Health Risk Manag. 2008; 4(5): 1141-1143, indexed in Pubmed: 19183765

6. Degenhardt K, Singh MK, Aghajanian H, et al. Semaphorin $3 d$ signaling defects are associated with anomalous pulmonary venous connections. Nat Med. 2013; 19(6): 760-765, doi: 10.1038/nm.3185, indexed in Pubmed: 23685842.

7. ElBardissi AW, Dearani JA, Suri RM, et al. Left-sided partial anomalous pulmonary venous connections. Ann Thorac Surg. 2008; 85(3): 1007-1014, doi: 10.1016/j. athoracsur.2007.11.038, indexed in Pubmed: 18291189.
8. Etienne D, John A, Menias CO, et al. Annular pancreas: a review of its molecular embryology, genetic basis and clinical considerations. Ann Anat. 2012; 194(5): 422-428, doi: 10.1016/j.aanat.2012.04.006, indexed in Pubmed: 22694842.

9. Fernandes-Silva H, Correia-Pinto J, Moura RS. Canonical sonic hedgehog signaling in early lung development. J Dev Biol. 2017; 5(1), doi: 10.3390/jdb5010003, indexed in Pubmed: 29615561.

10. Hamm M, Röttger $P$, Fiedler $C$. [Pancreas anulare as a rare differential diagnosis of duodenal stenosis in adulthood]. Langenbecks Arch Chir. 1997; 382(6): 307-310, doi: 10.1007/s004230050072, indexed in Pubmed: 9498201.

11. Haramati LB, Moche IE, Rivera VT, et al. Computed tomography of partial anomalous pulmonary venous connection in adults. J Comput Assist Tomogr. 2003; 27(5): 743-749, doi: 10.1097/00004728-200309000-00011, indexed in Pubmed: 14501365.

12. Healey J. An anatomic survey of anomalous pulmonary veins: their clinical significance. J Thorac Surg. 1952; 23(5): 433-444, doi: 10.1016/s0096-5588(20)31117-x.

13. Kim JiH, Jin ZWu, Murakami G, et al. Persistent right umbilical vein: a study using serial sections of human embryos and fetuses. Anat Cell Biol. 2018; 51(3): 218-222, doi: 10.5115/acb.2018.51.3.218, indexed in Pubmed: 30310717.

14. Kiseleva IP, Malsagov GU. Differential diagnosis of anomalous pulmonary venous return. A clinical-roentgenological study. Cor Vasa. 1984; 26(2): 140-146, indexed in Pubmed: 6734216.

15. Knox S, Madruga M, Carlan SJ. Rare congenital aberrant left superior pulmonary vein discovered with central line placement in a patient with critical cardiorespiratory collapse. Case Rep Pulmonol. 2017; 2017: 8728904, doi: 10.1155/2017/8728904, indexed in Pubmed: 29057135.

16. Koyama K, Sano Go, Hata Y, et al. An anomalous unilateral single pulmonary vein associated with a bone morphogenetic protein receptor II gene mutation. Intern Med. 2014; 53(5): 461-466, doi: 10.2169/internalmedicine.53.9245, indexed in Pubmed: 24583436.

17. Lapa T, Vedelago J, Kim H, et al. An unusual constellation of congenital malformations in a single patient including partial anomalous pulmonary venous return, persistent left superior vena cava, aberrant pulmonary fissure, anomalous aortic arch, tracheal diverticulum and annular pancreas. BMJ Case Rep. 2014; 2014, doi: 10.1136/bcr2012-008014, indexed in Pubmed: 25362184.

18. Low JYL, Nardini M, Zirafa C, et al. A surprise aberrant pulmonary vein. Interact Cardiovasc Thorac Surg. 2018; 26(2): 367, doi: 10.1093/icvts/ivx309, indexed in Pubmed: 29049785.

19. Minami K, Boshi H, Minami T, et al. 1 p36 deletion syndrome with intestinal malrotation and annular pancreas. Eur J Pediatr. 2005; 164(3): 193-194, doi: 10.1007/s00431004-1581-z, indexed in Pubmed: 15717182.

20. Raisher BD, Dowton SB, Grant JW. Father and two children with total anomalous pulmonary venous connection. Am J Med Genet. 1991; 40(1): 105-106, doi: 10.1002/ajmg.1320400122, indexed in Pubmed: 1887837.

21. Snellen HA, van Ingen HC, Hoefsmit EC. Patterns of anomalous pulmonary venous drainage. Circulation. 1968; 38(1): 45-63, doi: 10.1161/01.cir.38.1.45, indexed in Pubmed: 11712292.

22. Subotich D, Mandarich D, Milisavljevich M, et al. Variations of pulmonary vessels: some practical implications for lung resections. Clin Anat. 2009; 22(6): 698-705, doi: 10.1002/ ca.20834, indexed in Pubmed: 19637303.

23. Whelan TJ, Hamilton GB. Annular pancreas. Ann Surg. 1957; 146(2): 252-262, doi: 10.1097/00000658195708000-00015, indexed in Pubmed: 13459275.

24. White JJ, Skinner GB, MacLean LD. Hepatoportography via the umbilical vein: a superior approach to diagnosis in liver disease. Can Med Assoc J. 1966; 95(20): 997-1003, indexed in Pubmed: 5924949. 
